\title{
EVALUATION OF THE EFFECT OF SURGICALLY ASSISTED RAPID MAXILLARY EXPANSION ON TEMPOROMANDIBULAR JOINT DISC POSITION WITH MAGNETIC RESONANCE IMAGING*
}

\begin{abstract}
Objective: To evaluate, by magnetic resonance 1maging (MRI), the effects of surgically assisted rapid maxillary expansion (SARME) on the temporomandibular joint (TMJ) disc position.
\end{abstract}

Methods: Patients with maxillary transversial discrepeancies treated SARME analyzed prospectively. The magnetic resonance imaging assessments of the TMJ were obtained before SARME operation and after expansion process. Retention period, gender and presence of wisdom teeth were the predictor variables. Disc position index (DPI) values were calculated and analyzed as an outcome variable.

Results: The study included 13 subjects ( 4 male, 9 female) with a mean age of $19.5 \pm 2.3$ years. After treatment there was excess changing position seen in three articular disc relative the condyle in three patient. Retention period, gender and presence of wisdom teeth were not significantly effected TMJ disc in terms of DPI values in mouth opened or closed position $(\mathrm{p}>0.05)$.

Conclusion: According to our study TMJ disc position was not effected significantly by SARME ( $\mathrm{p}>0.05)$.

Keywords: Surgically assisted rapid maxillary expansion, Temporomandibular joint, Magnetic resonance imaging.

*This study was previously presented as an oral presentation at AÇBID 13th International Congress with EACMFS Endorsement held in Antalya, Turkey on April 26, 2019.
Murat Kaya ${ }^{1}$

iD * Mehmet Fatih Şentürk ${ }^{2}$

Yavuz Findık ${ }^{3}$

ORCID IDs of the authors:

M.K. 0000-0001-8269-930X

M.F.Ş. $\quad$ 0000-0001-8611-281X

Y.F. $\quad$ 0000-0003-3483-3177

1 Department of Oral and Maxillofacial Surgery, Erzincan, Turkey.

2 Department of Oral and Maxillofacial Surgery, Faculty of Dentistry, Ankara Yıldırım Beyazıt University, Ankara, Turkey.

3 Department of Oral and Maxillofacial Surgery, Faculty of Dentistry, Süleyman Demirel University, Isparta, Turkey.

$\begin{array}{ll}\text { Received } & : 24.10 .2020 \\ \text { Accepted } & : 17.05 .2021\end{array}$

How to Cite Kaya M, Şentürk MF, Fındık Y. Evaluation of Effect of Surgically Assisted Rapid Maxillary Expansion Surgery on The Temporomandibular Joint Disc Position with Magnetic Resonance Imaging. Cumhuriyet Dent J 2021;24:4:318-325.

*Corresponding Author:

Department of Oral and Maxillofacial Surgery, Faculty of Dentistry, Ankara Yıldırım Beyazıt University, Ayvalı 150.sk, Etlik / Ankara, Turkey.

Phone: + 905309513687

E-mail: fatih.senturk84@ gmail.com 


\section{INTRODUCTION}

Transverse discrepancies are common problems in adolescents and adults with orthodontic disorders. Transverse maxillary discrepancies can be skeletal and/or dental, and clinically, they are seen as a unilateral or bilateral posterior cross-bite. ${ }^{1,2} \mathrm{~A}$ surgically assisted rapid maxillary expansion (SARME) is the preferred treatment for transverse maxillary discrepancies in individuals with completed growth who have transverse discrepancies of more than $5 \mathrm{~mm}^{2}$ Whether or not a morphological or functional interaction on the temporomandibular joint (TMJ), either directly or indirectly, is caused by a surgical intervention to change the position of the jaws is a suggestive parameter.

Magnetic resonance imaging (MRI) is the gold standart to determine the chewing muscles, morphology, disc position and pathologies, bone structures, retrodiscal tissues, posterior attachment, inflammatory diseases of the joint (and the soft tissue changes caused by them), and any postoperative changes can be determined. Previous cadaver studies have indicated that MRI exhibited $95 \%$ accuracy in the evaluation of the disc location and disc form, and 93\% accuracy in the evaluation of the structural changes of the bone. ${ }^{3}$ The standard TMJ MRI protocol contains parallel, oblique, coronal, and oblique sagittal images parallel to the long axis of the condylar head. The sagittal images should be obtained with the mouth in both closed and open positions in order to determine the disc movements.

A systematic review ${ }^{4}$ evaluating conventional rapid maxillary expansion in growing patients showed that there is no change in the position and shape of the articular disc immediately after expansion. However, there is no evidence available on the subject when using, in adults, the rapid expansion of the surgically assisted maxilla.

The aim of this study was to evaluate, by MRI, the effects of the SARME on the TMJ disc position. We hypoestesized that occlusal changes after SARME could effect the TMJ and disc. Spesifically gender, wisdom tooth presence and retention period could rate the effectivity.

\section{METHODS}

The subjects included in this study were selected from the patients who presented to the Orthodontics Department of the Faculty of Dentistry at Suleyman Demirel University for orthodontic treatment between 2016 and 2018. Ethical approval was obtained from the local Ethics Committee (Date/Number: 04.11.2015/212).

The patient inclusion criteria were as follows:

- Skeletal transverse maxillary discrepancy with a unilateral or bilateral cross-bite.

- No congenital craniofacial deformities (cleft palate, syndromes, etc.).

- No surgical procedures performed in the upper or lower jaws.

- Non-growing patients with bone maturation evidence by hand and wrist radiography.

- The patient exclusion criteria were as follows:

- Lack of pre-treatment and/or post-treatment records.

- Closed-field phobia.

- Artifacts in the MRI images that prevent disc evaluations.

All of the patients had appropriate skeletal development based on the results of hand-wrist films, skeletal transverse maxillary discrepancies and surgical maxillomandibular transverse difference index values $(>5 \mathrm{~mm})$, as measured in postero-anterior radiographs. For the maxillary expansion in each of the patients, a dental support device with a Hyrax screw was placed parallel to the midline by the orthodontist one or a few days before the operation. It was cemented with glass ionomer cement via banding to the first premolar and first molar teeth.

\section{Surgery and Expansion Protocol}

In order to ensure standardization, all of the operations were performed under general anesthesia according to the asepsis and antisepsis rules using the same technique by the same team in the Maxillofacial Surgery Department of Suleyman Demirel University. All of the patients underwent bilateral Le Fort I osteotomies with piezoelectric surgeries. Midpalatal suture separations were conducted in all of the patients without pterygomaxillary osteotomies. In all of 
the patients, the Hyrax was activated until a $1 \mathrm{~mm}$ midline diastema was achieved in order to ensure symmetrical bone expansion. No complications were seen in any of the patients.

After a latency period of 5 days, the expansions were performed at a rate of 0.5 $\mathrm{mm} /$ day for 21 days, with no over corrections. Then, the appliance was stabilized for 4 or 5 months (retention period).

\section{Radiological Evaluation}

All of the MRI images were evaluated by 2 specialist radiologists and the image evaluations were based on the criteria of Tasaki and Westesson (3). In the sagittal plane, with a mouth closed position, the TMJ disc position was considered to be normal if the posterior band was located at the between 11 and 12 o'clock relative to the condyle. After that, the numerical measurements were performed using the same slice thickness that was used with the disc position index (DPI).

\section{DPI}

The articular disc position was assessed using a modification of the method used by Vargas Pereira. ${ }^{5}$ The following reference points and measuring variables were used (Figure 1):

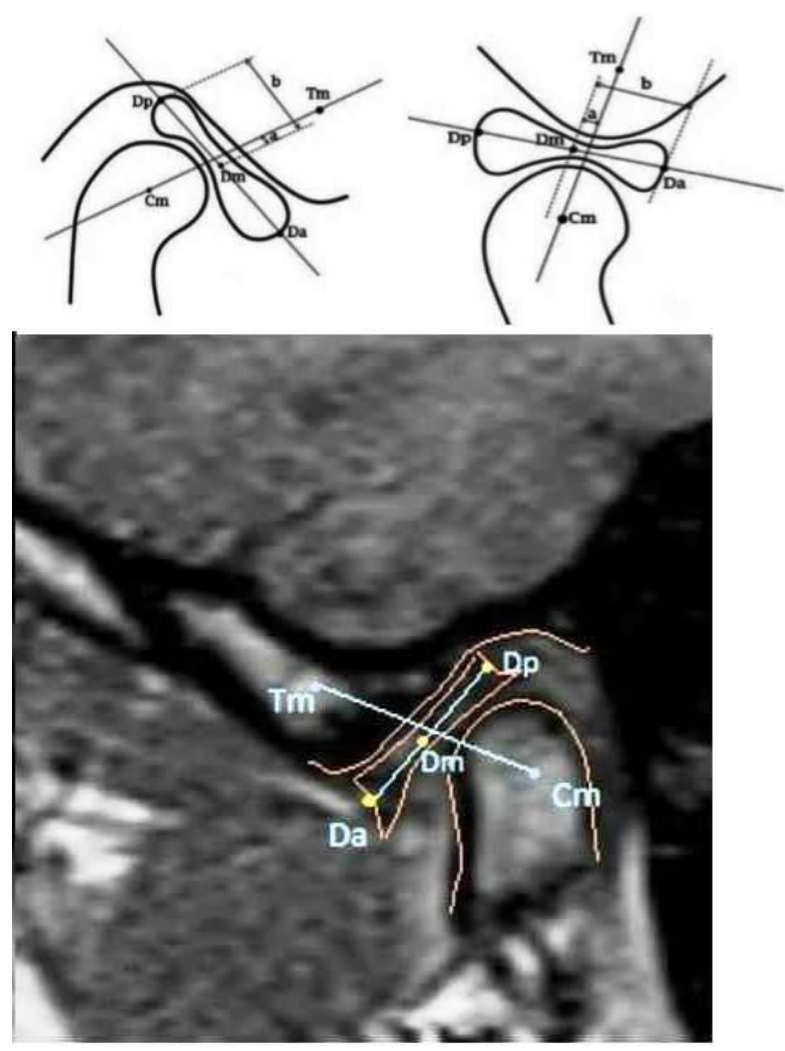

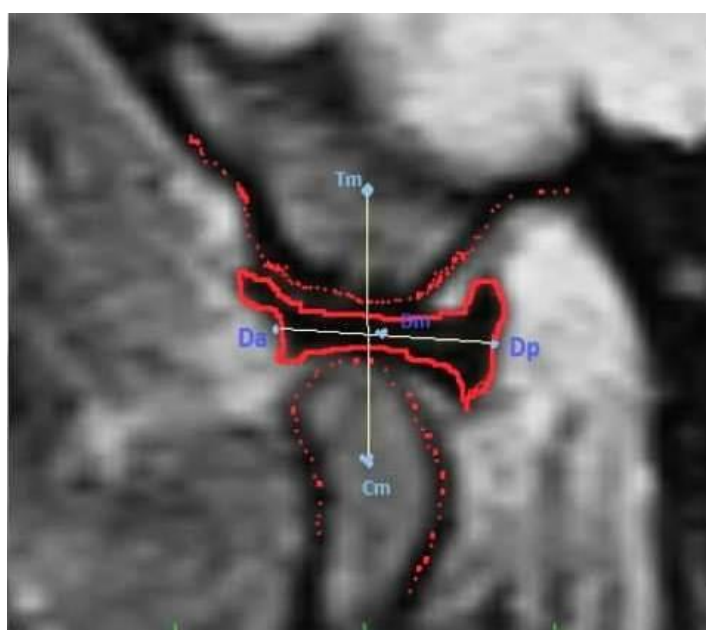

Figure 1.Reference points used for the disc position index in the mouth closed and mouth open positions in MRI.

$\mathrm{Cm}$ : Midpoint of the mandibular condylar head (as assessed by visual inspection).

Tm: Midpoint of the tuberculum articulare (as assessed by visual inspection).

Da: Anterior point of the articular disc.

Dp: Posterior point of the articular disc.

Dm: Midpoint of the articular disc (the midpoint of line Da-Dp).

a: Position of the articular disc as defined by the distance from the Dm (on line Da-Dp) to line CmTm.

b: Half the length of the articular disc as defined by the distance (Da-Dp).

For the final assessment of the disc position, the disc position index was calculated as follows:(a:b) $\times 100$.

The DPI describes the position of the articular disc in relation to the mandibular condyle and the temporal articular eminence. In a centered disc position, the midpoint of the disc (Dm) is on line $\mathrm{Cm}-\mathrm{Tm}$, and distance $\mathrm{a}$ is zero. In a protrusive disc position, the Dm is in front of line $\mathrm{Cm}-\mathrm{Tm}$, and distance a is positive. In a retrusive disc position, the Dm is behind line $\mathrm{Cm}-\mathrm{Tm}$, and distance a is negative. ${ }^{6}$

In order to standardize the DPIs of all of the TMJs in the mouth open and mouth closed positions, the measurements were performed 3 times by two radiologists, with one surgeon observing, and the means of the DPI measurements were used. 


\section{Variables}

The predictor variable was the retention period, gender and presence of wisdom teeth. TMJ disc position change values after SARME evaluated by DPI was the outcome variable. of the study. Demographical and clinical data of the patients were obtained from the forms collected in patients files.

\section{Statistical Evaluation}

Data were analyzed using IBM SPSS Statistics for Windows (version 21.0; IBM Corp., Armonk, NY, USA. For the DPI, the arithmetic mean and the standard deviation were calculated. The Mann-Whitney U test was used for the comparisons between two groups, and the Kruskal-Wallis $\mathrm{H}$ test was used for the comparisons between four groups. The level of significance was set to $\mathrm{p}<0.05$.

\section{RESULTS}

13 patients included in this study ( 9 females and 4 males), and all of them were older than 17 years. All of the patients had bilateral cross-bites and no history of bruxism. 6 patient's wisdom third molars are absent and remaining 7 was not extracted in SARME operation. In terms of retention period 8 patient was consolidated 4 months whereas 5 was consolidated 5 months to prevent relapse.

After evaluating the MRI images, it was determined that 4 patients had anterior disc displacements before the treatment. However, after the expansion treatment, there were disc position changes in only three patients (Table 1).

Table 1. Preoperative and post retention measurements in the mouth open and mouth closed positions.

\begin{tabular}{|c|c|c|c|c|c|c|c|c|}
\hline \multirow{3}{*}{ Patient } & \multicolumn{4}{|c|}{ Mouth closed } & \multicolumn{4}{|c|}{ Mouth open } \\
\hline & \multicolumn{2}{|c|}{ Preoperative } & \multicolumn{2}{|c|}{ Post-retention } & \multicolumn{2}{|c|}{ Preoperative } & \multicolumn{2}{|c|}{ Post-retention } \\
\hline & Right & Left & Right & Left & Right & Left & Right & Left \\
\hline $1 *$ & +191.6 & -19.6 & +22.5 & -19 & +6 & -13.3 & 0 & -9.1 \\
\hline 2 & -13.9 & +0.4 & -13.9 & +0.4 & -9.3 & 0 & -15.9 & +0.6 \\
\hline 3 & -2.5 & -2.9 & -5.9 & 0 & -6.6 & 0 & -6.2 & 0 \\
\hline 4 & +3 & +2 & +4 & 0 & 0 & 0 & +5.1 & +4 \\
\hline 5 & +3 & +6.3 & -7 & +5 & 0 & 0 & -6.4 & +5.2 \\
\hline 6 & 0 & 0 & 0 & -7.9 & -7.1 & -0.1 & -5.3 & 0 \\
\hline $7 *$ & 0 & -1 & +6.2 & +56.25 & +6.9 & +4.3 & +2.5 & -2.3 \\
\hline 8 & +156.9 & +0.4 & +156.1 & +0.4 & +156.7 & -8.9 & +149.2 & -9.3 \\
\hline $9 *$ & +120 & +6.5 & +123.6 & +116.6 & 128.8 & -6.7 & +128.8 & -1.8 \\
\hline 10 & 0 & +0.2 & 0 & +0.2 & 0 & +5.8 & -7.5 & +9.4 \\
\hline 11 & +9 & +4.1 & +7.1 & +1 & -6.6 & -0.1 & 0 & +1.7 \\
\hline 12 & $-4,7$ & +100 & $-11,3$ & +100 & $+0,1$ & 0 & 0 & $+6,6$ \\
\hline 13 & -3.7 & -5.4 & +4.2 & +3.5 & 0 & 0 & +2.3 & +1.7 \\
\hline
\end{tabular}

There were no significant differences between the gender, retention periods and wisdom tooth extractions in terms of value changes between the right and left TMJ DPI values in the mouth open and mouth closed positions ( $\mathrm{p}>0.05)$ (Table 2-4).

Table 2. Left and right differences according to gender (Sd:Standart deviation).

\begin{tabular}{|c|c|c|c|c|c|c|}
\hline \multirow{2}{*}{ Gender } & & \multicolumn{2}{|l|}{ Group } & \multicolumn{3}{|c|}{ Kruskal-Wallis H test } \\
\hline & & $\bar{n}$ & Mean \pm Sd & Average & $\mathbf{H}$ & $\mathbf{P}$ \\
\hline \multirow{4}{*}{ Right \% difference } & Mouth closed-female & 9 & $22 \pm 55.3$ & 13.61 & \multirow{4}{*}{0.264} & \multirow{4}{*}{0.967} \\
\hline & Mouth closed-male & 4 & $3.1 \pm 2.3$ & 11.75 & & \\
\hline & Mouth open-female & 9 & $4.2 \pm 3.2$ & 14.06 & & \\
\hline & Mouth open - male & 4 & $4.1 \pm 2.6$ & 13.75 & & \\
\hline \multirow{4}{*}{ Left $\%$ difference } & Mouth closed-female & 9 & $14.31 \pm 36.09$ & 11.5 & \multirow{4}{*}{1.4} & \multirow{4}{*}{0.704} \\
\hline & Mouth closed-male & 4 & $16.31 \pm 2.73$ & 16.75 & & \\
\hline & Mouth open-female & 9 & $3.03 \pm 2.39$ & 14.11 & & \\
\hline & Mouth open- male & 4 & $3.1 \pm 2.85$ & 13.38 & & \\
\hline
\end{tabular}


Table 3. Left and right differences according to the retention period ( $\mathrm{Sd}$ :Standart deviation).

\begin{tabular}{|c|c|c|c|c|c|c|}
\hline \multirow{2}{*}{\multicolumn{2}{|c|}{ Retention period distribution }} & \multicolumn{2}{|c|}{ Group } & \multicolumn{3}{|c|}{ Kruskal-Wallis H test } \\
\hline & & $\mathbf{n}$ & Mean+Sd & Average & $\mathbf{H}$ & $\mathbf{p}$ \\
\hline \multirow{4}{*}{ Right $\%$ difference } & Mouth closed-4 months & 8 & $23 \pm 59.1$ & 10.69 & \multirow{4}{*}{3.7} & \multirow{4}{*}{0.291} \\
\hline & Mouth closed-5 months & 5 & $5.2 \pm 2.4$ & 16.8 & & \\
\hline & Mouth open-4 months & 8 & $5.2 \pm 2.7$ & 16.13 & & \\
\hline & Mouth open-5 months & 5 & $2.7 \pm 2.8$ & 10.5 & & \\
\hline \multirow{4}{*}{ Left $\%$ difference } & Mouth closed-4 months & 8 & $1.84 \pm 2.67$ & 9.69 & \multirow{4}{*}{5.6} & \multirow{4}{*}{0.131} \\
\hline & Mouth closed-5 months & 5 & $35.87 \pm 47.58$ & 18.6 & & \\
\hline & Mouth open- 4 months & 8 & $2.26 \pm 2.18$ & 11.94 & & \\
\hline & Mouth open-5 months & 5 & $4.32 \pm 2.45$ & 17 & & \\
\hline
\end{tabular}

Table 4. Left and right differences according to the wisdom tooth status (Sd:Standart deviation).

\begin{tabular}{|c|c|c|c|c|c|c|}
\hline \multirow{2}{*}{ Wisdom tooth } & & \multicolumn{2}{|l|}{ Group } & \multicolumn{3}{|c|}{ Kruskal-Wallis H test } \\
\hline & & $\bar{n}$ & Mean+Sd & Average & $\mathbf{H}$ & $\mathbf{p}$ \\
\hline \multirow{4}{*}{ Right $\%$ difference } & Mouth closed-no tooth & 6 & $30.3 \pm \overline{6} 8.1$ & 12.17 & \multirow{4}{*}{3.3} & \multirow{4}{*}{0.339} \\
\hline & Mouth closed-wisdom tooth & 7 & $4.1 \pm 3.0$ & 13.79 & & \\
\hline & Mouth open-no tooth & 6 & $6 \pm 2.1$ & 18 & & \\
\hline & Mouth open-wisdom tooth & 7 & $2.7 \pm 2.7$ & 10.5 & & \\
\hline \multirow{4}{*}{ Left $\%$ difference } & Mouth closed-no tooth & 6 & $2.15 \pm 3.04$ & 10.42 & \multirow{4}{*}{1.5} & \multirow{4}{*}{0.668} \\
\hline & Mouth closed-wisdom tooth & 7 & $25.88 \pm 42.43$ & 15.43 & & \\
\hline & Mouth open-no tooth & 6 & $2.55 \pm 2.10$ & 13.17 & & \\
\hline & Mouth open-wisdom tooth & 7 & $3.49 \pm 2.75$ & 14.5 & & \\
\hline
\end{tabular}

\section{DISCUSSION}

Although skeletal maturation is completed earlier in female patients, skeletal development continues chronologically in male patients for a while longer. Therefore, rapid maxillary expansion treatments in male patients can be continued at a later age when compared to female patients. ${ }^{2}$ In the literature, the incidence of TMJ disorders is reported to be higher in females ${ }^{7}$ In our study most of the patients were female however be based on gender distribution TMJ disc position was not changed statistically significant in terms of DPI values ( $p>0.05$ ).

Many researchers have reported that their patients had TMJ disorder signs and symptoms of various degrees before undergoing orthognathic surgery. Laskin et al. ${ }^{8}$ reported that $14 \%$ of the patients undergoing orthognathic surgery had symptoms and signs of TMJ disorders. Kerstens et $a l^{9}$ reported that of 480 patients with dentofacial deformities, $16 \%$ had TMJ disorder symptoms before surgery. However, Link and Nickerson found the incidence of TMJ internal irregularities to be very high $(97 \%)$ in their orthognathic surgery population. ${ }^{10}$ In our study, it was determined that 4 of the 13 patients already had anterior disc displacement in their TMJs before treatment. However, based on the number of patients in our study, in order to determine the TMJ internal irregularity rate in patients with transverse maxillary discrepancies, we suggest that a multi-sample study be performed to reveal the statistical findings.

Studies investigating the effects of orthognathic surgery on the TMJ disc have been performed mainly on sagittal split ramus osteotomy (SSRO), vertical ramus osteotomy, and double jaw osteotomy procedures that were combined with Le Fort I osteotomies. In their study of patients undergoing intraoral vertical ramus osteotomy (IVRO) or SSRO procedures with or without the combined use of a Le Fort I procedure, Ueki et al. reported that the mandibular condyles were displaced in the anterio-inferior direction after the osteotomy in the patients undergoing IVROs. In the MRI examinations of the TMJ discs, they found that the discs with normal positions were not affected, but the discs with anterior displacement had significantly improved. In the patients with mandibular prognathism who underwent SSROs, no significant changes were observed in the TMJ disc positions according to the MRI findings 6 months after the orthognathic surgery, but the TMJ symptoms had improved. ${ }^{11-13}$ In addition to the changes in the three-dimensional positions of 
the jaws in space, we can see that the bad interdigitation became good interdigitations after the orthognathic surgery. Consequently, this condition led to an improvement in the TMJ symptoms due to occlusal healing, and thus, a favorable condition in terms of intra-TMJ irregularities. However, interdigitation is present after a SARME is impaired, and this leads to interference, which may affect the TMJ during functional movements, such as the occlusal morphology, chewing, and biting. However, when we considered our findings, we observed that the SARME had no significant effects on the TMJ disc position within a certain period of time.

The clockwise rotation of the mandible is a major effect of SARME on the mandible, although there is no consensus about the amount or stability of this change. ${ }^{14,15-18}$ Altug-Atac et $a l .{ }^{14}$ and Gunbay et $a l .{ }^{16}$ reported a clockwise rotation of the mandible after SARME, while Iodice et $a{ }^{17}{ }^{17}$ and Parhiz et al. ${ }^{19}$ reported no significant change in the rotational movement of the mandible. These changes are presented with the downward rotation of the menton point. In fact, there are differences in the time intervals for the evaluations of the mandibular rotations in these studies. However, according to the results, if the mandibular rotation is based on the tendency to go back to the preoperative position 6 months after the SARME, the motion is a temporary movement. ${ }^{18}$ The methodological differences between these studies and evaluations at the different time points confirm the differences in their mandibular rotation findings.

In our study, according to the retention period (4 or 5 months) there were no significant differences in terms of the DPI values in mouth opened or closed position ( $p>0.05$ ). These periods correspond to the time during which the mandible moves to its original position. Therefore, taking this parameter into consideration, in another study, the mandibular rotation can be confirmed using cephalometric films, and the MRI images can be taken at an earlier time following the expansion; then, the disc position evaluations can be performed.

Some of the factors that may affect the mandibular position, functional forces, and occlusal relationship are the shape, extent, and extent of enlargement of the maxillary segments. Kilıç et al. ${ }^{20}$ stated that the tipping of the alveolar segments was higher and the decrease in the palate depth was greater in the group without pterygoid separation. Ferraro et al. ${ }^{21}$ examined the effects of pterygoid separation in a study using cone beam computed tomography, and they reported that the same expansion amount was obtained with or without pterygoid separation. The expansion was greater in the molar region in both groups, but the dentoalveolar tipping amount was greater if no pterygoid separation was performed. In our study, a non invasive technique without pterygoid separation was used. Therefore, there was a more rotational opening and occlusal template change. It is also possible to say the opposite. The effects of a pterygoid separation on the occlusal morphology or the movement of the mandible, indirectly, on the TMJ components can be considered in a different study.

In the literature, TMJ disease has been shown as a complication caused by the surgical extraction of the wisdom teeth, and there are different studies on this subject. In 2002, Huang et $a l .^{7}$ examined the risk factors causing painful TMJ disorders, and they collected these factors under subgroups, such as bruxism, facial trauma, third molar extraction, orthodontic treatments, frequent dental treatments, female gender, depression, and psychogenic disorders. When opening the mouth, the ligaments forming the TMJ are stretched considerably. Traumatic factors, such as intubation, wisdom tooth extraction, and longlasting dental treatments can also cause the ligaments to be affected, with the disc motion becoming irregular and joint dysfunction starting. In our study, we divided the patients into two groups, those who underwent wisdom tooth extraction surgery before the SARME and those who did not. Thus, in this procedure, which can be considered to be a macro-trauma in terms of the TMJ, we tried to see the effects of the TMJ disc on the MRI findings after the upper jaw expansion. However, according to the wisdom tooth presence in SARME no significant 
difference was found in terms of DPI values $(\mathrm{p}>0.05)$.

\section{CONCLUSIONS}

Be based on the small sample size used in our study, SARME was not effected the TMJ disc position. Gender, retention period, and wisdom tooth presence are not effected the TMJ disc position in terms of DPI's after SARME. There were no complaints of bruxism based on the clinical histories of the patients. However, no objective diagnosis of bruxism was obtained. For this reason, large sample studies are needed to compare the disc change incidences according to the morphological differences in the MRI images. The samples should also be grouped according to the different surgical techniques.

\section{FUNDING}

This study was supported by Suleyman Demirel University Projects of Scientific Investigation commission with the project number of 4684DU1-16.

\section{ACKNOWLEDGEMENTS}

We are grateful to "İstatistik Dünyası" for their statistical assistance and Radiology Department of Medicine and Dentistry Faculty of Suleyman Demirel University for their radiological assessments and Anesthesiology Department of Suleyman Demirel University Dentistry Faculty for their assistance in general anesthesia.

\section{REFERENCES}

1. da Silva Filho OG, Boas MC, CapelozzaFilho L. Rapid maxillary expansion in the primary and mixed dentitions: a cephalometric evaluation. Am J Orthod Dentofacial Orthop 1991;100(2):171-179.

2. Suri L, Taneja P. Surgically assisted rapid palatal expansion: a literature review. Am J Orthod Dentofacial Orthop 2008;133(2):290-302.

3. Tasaki MM, Westesson PL. MR imaging of the temporomandibular joint: diagnosis accuracy with sagittal and coronal images. Radiology 1993;186(3):723-729.

4. Torres D, Lopes J, Magno MB, Maia LC, Normando D, Leão PB; Effects of rapid maxillary expansion on temporomandibular joints: A systematic review. Angle Orthod 2020;90(3):442-456.

5. Vargas Pereira MR. Quantitative Auswertungen bildgebender Verfahren und Entwicklulng einer neuen metrischen Analyse für Kiefergelenkstrukturen im Magnet resonanz-tomogramm (thesis). Kiel, Germany:University of Kiel:1997.

6. Pancherz H, Ruf S, Thomalske-Faubert C. Mandibular articular disc position changes during Herbst treatment: a prospective longitudinal MRI study. Am J Orthod Dentofac Orthop 1999;116(2):207214.

7. Huang GJ, Leresche L, Critchlow CW, Martin MD, Drangsholt MT. Risk factors for diagnostic subgroups of painful temporomandibular disorders (TMD). J Dent Res 2002;81(4):284-288.

8. Laskin DM, Ryan WA, Greene CS. Incidence of temporomandibular symptoms in patients with major skeletal malocclusions: a survey of oral and maxillofacial surgery training programs. Oral Surg Oral Med Oral Pathol Oral Radiol Endod 1986;61(6):537-541.

9. Kerstens HC, Tuinzing DB, van der Kwast WA.Temporomandibular joint symptoms in orthognathic surgery. J Craniomaxillofac Sur. 1989;17(5):215-218.

10.Link JJ, Nickerson JW Jr. Temporomandibular joint internal derangements in an orthognathic surgery population. Int $\mathrm{J}$ Adult Orthod Orthognath Surg 1992;7(3):161-169.

11.Ueki K, Marukawa K, Shimada M, Yoshida K, Hashiba Y, Shimizu C, et al. Condylar and disc positions after intraoral vertical ramus osteotomy with and without a Le Fort I osteotomy. Int J Oral Maxillofac Surg 2007;36(3):207-213.

12.Ueki K, Marukawa K, Shimada M, Hashiba Y, Nakgawa K, Yamamoto E. Condylar and disc positions after sagittal split ramus osteotomy with and without Le Fort I osteotomy. Oral Surg Oral Med Oral Pathol Oral Radiol Endod 2007;103(3):342-348.

13.Ueki K, Marukawa K,Nakagawa K,Yamamoto E. Condylar and temporomandibular joint disc positions after mandibular osteotomy for prognathism. J Oral Maxillofac Surg 2002;60(12):1424-1432.

14. Altuğ-Ataç AT, Karasu HA, Aytaç D. Surgically assisted rapid maxillary expansion compared with orthopedic rapid maxillary expansion. Angle Orthod 2006;76(3):353-359.

15. Bretos JL, Pereira MD, Gomes HC, Toyama Hino C, Ferreira LM. Sagittal and vertical maxillary effects after surgically assisted rapid maxillary expansion 
(SARME) using Haas and hyrax expanders. J Craniofac Surg 2007;18(6):1322-1326.

16.Gunbay T, Akay MC, Gunbay S, Aras A, Koyuncu BO, Sezer B. Transpalatal distraction using boneborne distractor: clinical observations and dental and skeletal changes. J Oral Maxillofac Surg 2008;66(12):25032514.

17. Iodice $\mathrm{G}$, Bocchino $\mathrm{T}$, Casadei M, Baldi D, Robiony M. Evaluations of sagittal and vertical changes induced by surgically assisted rapid palatal expansion. J Craniofac Surg2013;24(4):1210-1214.

18. Oliveira TFM, Pereira-Filho VA, Gabrielli MFR, Gonçales ES, Santos-Pinto A. Effects of surgically assisted rapid maxillary expansion on mandibular position: a three dimensional study. Prog Orthod 2017;18(1):22-27.

19.Parhiz A, Schepers S, Lambrichts I, Vrielinck
L, Sun Y, Politis C. Lateral cephalometry changes after SARPE. Int J Oral Maxillofac Surg 2011;40(7):662671.

20.Kilic E, Kilic B, Kurt G, Sakin C, Alkan A. Effects of surgically assisted rapid palatal expansion with and without pterygomaxillary disjunction on dental and skeletal structures: a retrospective review. Oral Surg Oral Med Oral Pathol Oral Radiol 2013;115(2):167174.

21.Ferraro-Bezerra $M$, Tavares $R N$, de Medeiros JR, Nogueira AS, Avelar RL, StudartSoares EC. Effects of pterygomaxillary separation on skeletal and dental changes after surgically assisted rapid maxillary expansion: a single-center, double-blind, randomized clinical trial. J Oral Maxillofac Surg 2018;76(4):844853. 